\title{
Pitch interactions in the perception of isolated musical triads
}

\author{
JOHN R. PLATT, RONALD J. RACINE, MARIANNA STARK, and MARGARET WEISER \\ McMaster University, Hamilton, Ontario
}

\begin{abstract}
In previous work done in our laboratory, we have investigated the perceived pitch class of isolated musical triads. We have found that as the amount of musical training increased, listeners' perceptions progress, from very confused percepts of pitch class, to analytic percepts corresponding to the pitch class of the highest note in the triad, and finally to synthetic percepts corresponding to the root note for the more harmonic triad types. In the present work, we used a pitch matching technique to determine the actual pitch, rather than merely the pitch class, perceived when listeners analytically "hear out" a particular note in a major triad. There was a strong tendency for the pitch of the analytically perceived note to be displaced by as much as 60 cents in the direction of the other notes in the triad. The magnitude of this effect decreased as musical training increased, and it was also affected by the relative salience of the individual triad notes. These results have implications for the mechanism of triad perception, and for claims regarding the harmonic equivalence of triad inversions.
\end{abstract}

The basic elements of harmony in Western music are triads or three-note chords produced by adding musical intervals of a third and a fifth above each note of the major and harmonic minor scales. The most common type of such chords are major triads, which add intervals of a major third and a perfect fifth above the root. There are numerous ways to voice a particular triad, because any of its three notes can be sounded in any octave. The position of the triad is defined by the pitch-height order of its notes. If the lowest note is the root, the triad is in root position. If the lowest note is the third or the fifth, the triad is in first or second inversion, respectively.

Both musical and psychoacoustic theories offer numerous suggestions as to how isolated triads are perceived. The psychological mechanisms of such perception are particularly interesting, since both types of theories suggest that various positions of a triad are harmonically equivalent, although they are different physical stimuli containing different internal musical intervals. As for musical theory, as early as the 18th century, Jean-Philippe Rameau (Rameau, 1750/1971) developed a theory of harmony based on the analysis of harmonics in stretched strings. He observed that all the frequencies in a major triad are integer multiples of what he called a "fundamental bass," which corresponds to the root note of the triad in a lower octave. Although Rameau was not aware of the phenomenon of residue or virtual pitch, by means of which a missing fundamental can be perceived from harmonic partials, his strong belief in octave equivalence allowed him to

This research was supported by Grants A8269 and A8629 from the Natural Sciences and Engineering Research Council of Canada. Reprints may be obtained from John R. Platt, Department of Psychology, McMaster University, 1200 Main Street West, Hamilton, Ontario L8S 4K1, Canada identify the fundamental bass with the root note of a triad and use it as the common factor supporting harmonic equivalence of different positions of the triad. Before that time, such variations in position were considered to be different chords.

In psychoacoustic theory, Terhardt $(1974,1984)$ has elaborated and provided a perceptual basis for Rameau's notion of the fundamental bass. According to Terhardt's virtual pitch theory, a major triad may produce any one of several different percepts. A listener may operate in an analytic perceptual mode, in which attention is selectively directed to the pitch of one or another of the notes in the triad. More commonly, however, the listener should operate in a synthetic perceptual mode, in which these individual pitches are processed further until they suggest a virtual pitch corresponding to the missing fundamental (fundamental bass) of the harmonic series formed by the triad's individual notes, and hence, to the pitch of the root note in a lower octave. The latter mode of processing is a type of pattern recognition allegedly acquired through early experience with the harmonic structure of speech and other harmonic complex sounds.

In most psychological research on chord perception, experimenters have either examined the perceived similarity of different chord types (major, minor, augmented, diminished) in order to determine the perceptual dimensions on which they vary (e.g., Roberts \& Shaw, 1984), or asked subjects to rate how well one chord follows another in an attempt to discover the cognitive structures relevant to tonality (e.g., Krumhansl, Bharucha, \& Kessler, 1982). As valuable as such studies are for other purposes, they offer little information about the pitches that subjects actually perceive when listening to chords.

Recently Platt and Racine (in press) have reported a series of experiments in which they investigated what pitch 
classes dominate subjects' perceptions of isolated musical triads. One technique consisted of a paired-comparisons task to determine which component pure tone of a triad sounded most similar to the triad. Musically inexperienced subjects showed no systematic preference, but subjects with a moderate amount of musical training consistently heard the highest pitched component tone as sounding most similar to the triad. Half of the professional musicians tested gave results similar to those given by the moderately experienced subjects, and the other half consistently heard a pure tone corresponding to the root note as sounding most similar to a major triad. This preference for the root note gradually shifted to a preference for the highest pitched component tone as the triad type became increasingly inharmonic. This last result was exactly what would be expected for the synthetic perceptual mode in virtual pitch theory, since inference of a virtual pitch corresponding to the missing fundamental is dependent on the extent to which the component tones are integer multiples of a common fundamental frequency.

A major question raised by Platt and Racine's (in press) paired-comparison results was why perceptual dominance by the pitch class of the root note in harmonic triad types was limited to a subset of the most highly trained musicians tested. The authors suggested that having subjects compare triads to pure tones may have biased them to an analytic perceptual mode and hence to dominance by the highest pitched note in the triad, rather than the root note. Helmholtz (1863) first suggested the importance of listening to a pure tone of the same pitch in order to direct attention to a particular partial of a complex tone, and Plomp (1976) has summarized more recent evidence for the importance of such a comparison in producing analytic perception. In order to avoid this problem, Platt and Racine (in press) employed a second technique, in which subjects vocally reproduced the pitch they heard when listening to a triad, and the pitch class of this vocalization was measured with a chromatic tuning meter. This technique produced results similar to those of the paired-comparisons task, except that dominance by the root note in harmonic triad types was much more widespread, and even occurred in some musically inexperienced subjects.

Although Platt and Racine's (in press) results give a clear picture of many of the factors and mechanisms determining the perceptually dominant pitch class for isolated musical triads, the techniques that they employed could only determine pitch class or note name, not exact pitch. A similar problem exists with respect to the fairly extensive psychoacoustic literature concerned with the ability to analytically hear out a particular partial in a complex tone, since such studies (e.g., Plomp, 1964; Pollack, 1964) typically involve a forced choice between a tone corresponding to the pitch of the partial in question and another tone whose pitch is maximally different from that of any partial present in the complex tone. Such a technique would not be sensitive to small variations in the analytically perceived pitch of the partial. The possibility thus remains that the exact pitch dominating perception of a triad is not independent of the other pitches present and hence of the position of the triad. In the case of synthetic perception of the pitch class of the root note, pitch interactions between the notes of the triad are an inherent part of pattern recognition models such as virtual pitch theory and are understood in some detail (Terhardt, Stoll, \& Seewann, 1982). However, Platt and Racine's (in press) results indicate that an analytic mode will prevail for less harmonic triad types and for less musically experienced subjects.

In the present experiments, we investigated the possible influence of other pitches present in a major triad on the analytically perceived pitch of a component note of the triad. The general technique involved the adjusting of the frequency of a pure tone over a range restricted to the pitch class of one of the component tones of a major triad so that the adjustable tone sounded maximally similar to the triad. The independent variables of major interest were the effect of the other two notes in the triad and the musical experience of the subject on this adjustment.

\section{EXPERIMENT 1}

In the first experiment in this series, the analytically perceived pitch of the root note of an A-major triad in root position was compared with that of the same root note in the first inversion of that triad formed by moving the third and fifth of the triad to the octave below the root. If these two pitches differ, that difference would have to be attributed to the placement of the third and the fifth above, rather than below, the root note, and hence the difference would represent pitch interactions between the component notes of the triad. Analytic perception of the root note of the triad was encouraged, by requiring the subjects to compare the triad to a pure tone whose range of adjustment was limited to a range of approximately plus or minus one semitone about the root note. The subjects also adjusted a pure tone to match the pitch of the same root note in the absence of the other two notes of the triad, as a control for time-order errors.

\section{Method}

Subjects. The subjects were 28 undergraduate students, ranging in age from 17 to 30 years. Five male students and 12 female students were classified as musically trained on the basis of self-reports of having had at least 3 years of formal musical training on one or more instruments other than percussion. Seven male students and 4 female students were musically untrained.

Apparatus. The experiment took place in a sound-attenuating room, with the subject seated in a comfortable chair in front of a table. An Apple II+ computer, equipped with a Mountain Computer Music System, controlled stimulus presentation and data collection. A Yamaha CA-410 audio amplifier conditioned the stimuli for binaural presentation through Realistic PRO-1 earphones. A Zenith ZVM-121 video monitor displayed instructions to the subjects, who adjusted the pitch of a comparison tone using a one-turn potentiometer with $1-\mathrm{Hz}$ resolution. The Mountain Computer Music System uses 8-bit digital-to-analog converters (DACs) driven at a 
sample rate of $31.25 \mathrm{kHz}$. The resulting analog signal was passed through a 15-kHz low-pass filter for smoothing. The 8-bit DACs were relatively noisy, having a signal-to-noise ratio of only $48 \mathrm{~dB}$.

Stimuli. The stimuli for this experiment consisted of isolated pure tones and of justly intoned, A-major triads composed of pure tones. Pure tones were used instead of the complex tones typical of musical instruments, in order to simplify interpretation of any pitch interactions found. Just intonation was used instead of the musically more common tempered scale, in order to preclude interpretation in terms of subjects attempting to correct mistuned consonances. In the case of major triads, however, the difference between just intonation and a tempered scale are slight, since the frequencies of the tonic notes are identical and those of the fifth differ by only 2 cents. The major difference occurs with respect to the major thirds, which still differ by only 14 cents.

Two positions of the triad with the same root note were employed as standard stimuli. The root-position triad was composed of A-880, $\mathrm{C} \#-1100$ and $\mathrm{E}-1320 \mathrm{~Hz}$. The first inversion triad consisted of $\mathrm{CH}-550, \mathrm{E}-660$, and A-880 $\mathrm{Hz}$. A third standard stimulus was a pure tone of the same frequency $(880 \mathrm{~Hz})$ as the root note of the triads. The comparison stimulus for all of these standards was a pure tone whose initial frequency on each trial was randomly offset $\pm 1 \%$ to $\pm 3 \%$ from the value $(880 \mathrm{~Hz})$ of the root note in the standard stimuli. The subjects could then adjust this initial frequency $\pm 6 \%$ to match the perceived pitch of the standard. Both standard and comparison stimuli had an envelope of 512-msec duration, including 10 -msec rise and fall times. Stimulus intensities were $60 \mathrm{~dB}$ SPL for triads and $62 \mathrm{~dB}$ SPL for isolated pure tones. These values served to roughly equate the loudness of the various stimuli.

Procedure. Each subject completed a total of 45 method-ofadjustments trials in a single session lasting approximately $1 \mathrm{~h}$. Each trial began with the subject centering the potentiometer knob with the aid of a numerical display on the video monitor and pushing a button. There were $512 \mathrm{msec}$ of standard stimulus, followed by $512 \mathrm{msec}$ of silence, and then $512 \mathrm{msec}$ of comparison stimulus. After 1 sec of silence, this sequence was repeated. The subject was instructed to adjust the pitch of the comparison stimulus until it matched that of the standard. The subject could make adjustments at any time, but the effect of an adjustment was not heard until the next onset of the comparison stimulus. This cycle continued until the subject pressed a button indicating a pitch match and ending the trial.

Each of the three standard stimuli was used on a random 15 of the 45 trials. On each trial, the computer recorded the identity of the standard stimulus, the initial frequency of the comparison stimulus, the number of standard-comparison pairs to which the subject listened before indicating a pitch match, and the final setting of the comparison stimulus. To facilitate evaluation of errors within a musical context, all comparison settings were transformed from Hertz to cents, where a cent is .01 semitone, and thus bears a logarithmic relationship to frequency. In the actual computation, the ratio of comparison to standard frequency was used, so that positive values indicated sharpening of the comparison with respect to the standard, and negative values indicated flattening.

\section{Results and Discussion}

Stimulus exposure. For each group of subjects and type of standard stimulus, Table 1 shows the mean number of standard-comparison pairs to which a subject listened before indicating a pitch match. These data are important for interpretation of the method-of-adjustments results, because stimulus exposure and number of adjustments were controlled by the subject. An analysis of variance indicated that musically trained subjects listened to slightly more pairs than did untrained subjects $[F(1,26)=4.82$, $p=.035]$, and that all subjects listened to fewer pairs when the standard stimulus was a pure tone than when it was a triad $[F(2,52)=22.22, p<.001]$. The interaction of these two variables did not approach statistical significance. The tendency for musically trained subjects to listen to more pairs will not be discussed, since it failed to replicate in subsequent experiments. Listening to fewer pairs when both standard and comparison stimuli were pure tones undoubtedly reflects the greater ease of pitch matching in this case (Platt \& Racine, 1985).

Relative errors. Table 2 shows the mean relative errors for the various groups and standard stimuli. A subject's relative error for a particular standard stimulus is simply the standard deviation of that subject's comparison settings, and it thus provides an index of individual consistency. An analysis of variance indicated that musically trained subjects were more consistent than untrained subjects $[F(1,26)=63.12, p<.001]$. In addition, settings were more consistent when both the standard and the comparison were pure tones than when the standard was a triad $[F(2,52)=22.72, p<.001]$; and this difference was largest for untrained subjects $[F(2,52)=7.57$, $p=.002]$. These findings are analogous to those of previous discrimination studies in which simple and complex tones were presented to subjects with varying levels of musical training (Platt \& Racine, 1985; Spiegel \& Watson, 1984).

Constant errors. The results of primary interest in Experiment 1 were constant errors. A constant error is simply the mean comparison setting; it reflects the perceived pitch of the standard in relation to that of the comparison stimulus. Table 3 shows the mean constant errors for the various groups and standard stimuli. When both standard and comparison stimuli were pure tones, constant errors were very small (less than 10 cents), indicating the lack of substantial time-order errors in the paradigm. When triads were used as standard stimuli, musically untrained subjects showed substantial pitch interactions. Thus, the comparison was set sharper than the root note for the root-

Table 1

Mean Number of Standard-Comparison Pairs to Which a Subject Listened Before Indicating a Pitch Match for the Various Groups and Standard Stimuli in Experiment 1

\begin{tabular}{cccc}
\hline & \multicolumn{2}{c}{ Standard Stimulus } & \\
\cline { 2 - 3 } Musical & $\begin{array}{c}\text { Root-Position } \\
\text { Training }\end{array}$ & $\begin{array}{c}\text { First-Inversion } \\
\text { Triad }\end{array}$ & $\begin{array}{c}\text { Root Note } \\
\text { Alone }\end{array}$ \\
\hline No & 11.0 & 12.8 & 6.4 \\
Yes & 15.8 & 14.2 & 10.2 \\
\hline
\end{tabular}

Table 2

Mean Relative Errors, Expressed as the Standard Deviation of a Subject's Comparison-Stimulus Settings in Cents, for the Various Groups and Standard Stimuli in Experiment 1

\begin{tabular}{cccc}
\hline & \multicolumn{2}{c}{ Standard Stimulus } & \\
\cline { 2 - 3 } Musical & $\begin{array}{c}\text { Root-Position } \\
\text { Training }\end{array}$ & $\begin{array}{c}\text { First-Inversion } \\
\text { Triad }\end{array}$ & $\begin{array}{c}\text { Root Note } \\
\text { Alone }\end{array}$ \\
\hline No & 41.6 & 40.7 & 25.4 \\
Yes & 12.1 & 15.8 & 9.3 \\
\hline
\end{tabular}


Table 3

Mean Constant Errors, Expressed as the Deviation in Cents of the Comparison Frequency from the Standard Frequency, for the Various Groups and Standard Stimuli in Experiment 1

\begin{tabular}{cccc}
\hline & \multicolumn{2}{c}{ Standard Stimulus } & \\
\cline { 2 - 3 } Musical & Root-Position & First-Inversion & Root Note \\
Training & Triad & Triad & Alone \\
\hline No & 27.5 & -60.2 & 8.0 \\
Yes & 22.9 & 10.2 & 3.4 \\
\hline
\end{tabular}

position triad, and considerably flatter than the root note for the first-inversion triad. Musically trained subjects, on the other hand, responded much more similarly to the two triad positions, setting the comparison stimulus slightly sharper than the root note in both cases. These differences were supported by an analysis of variance, which showed significant main effects for musical training $[F(1,26)=23.15, p<.001]$ and type of standard stimulus $[F(2,52)=50.00, p<.001]$, as well as a significant interaction between these two variables $[F(2,52)=$ 36.56, $p<.001]$.

The means shown in Table 3 are not the result of averaging over trials varying with respect to which note of the triad subjects used as a standard for adjusting the comparison stimulus. The restricted range of adjustment of the comparison stimulus did not allow it even to approximate any note in the triad other than the root. In addition, distributions of individual comparison-stimulus settings were consistently unimodal.

The finding that musically untrained subjects heard the pitch of the triads as deviating from the root note in the direction of the other notes in the triad is amenable to several different interpretations. In a number of previous studies (Greer, 1970; Lichte, 1941; Platt \& Racine, 1985), it has been reported that complex tones tend to be heard as being pitched slightly higher than simple tones of the same fundamental frequency, as if the pitch of the fundamental were being displaced slightly in the direction of the overtones. Such a pitch shift is compatible with the synthetic perceptual mode in Terhardt's $(1974,1984)$ virtual pitch theory. Both the magnitude and the direction of the shift have been shown to depend on factors such as the intensity of the complex tone, just as is predicted by the theory (Terhardt $\&$ Grubert, 1987).

In the present experiment, every effort was made to have the subjects perceive the root note of the triad analytically, rather than synthetically perceive the missing fundamental of the harmonic series formed by its notes. Assuming that these efforts were successful-an assumption that was tested in Experiment 2-it is reasonable to conclude that the pitch interactions shown by the musically untrained subjects reflect a general tendency for an analytically perceived pitch to be displaced in the direction of other spectral components present. An alternative interpretation is that the subject stores the individual pitch traces from the triad veridically, but that the subject is influenced by all of the pitch percepts stored when subsequently adjusting a pure tone to match the triad.
Whatever the mechanism of the pitch interactions observed for musically untrained subjects, trained subjects were able to avoid them and set the frequency of the comparison stimulus close to that of the root note of the triad. These results suggest a natural tendency to pitch interactions, which can be overcome with even limited amounts of musical training. The mechanism for this change appears to be a general improvement in analytic hearing, which is attributable to either a reduction in the influence of other spectral components on a particular analytic pitch percept, or an improved ability to ignore other pitch percepts produced by the standard when adjusting the comparison stimulus.

\section{EXPERIMENT 2}

Every effort was made in Experiment 1 to have the subjects analytically perceive the root note of the triad. However, it would also be possible to adjust a comparison tone in the frequency range of the root note on the basis of octave equivalence with a synthetically perceived missing fundamental of the harmonic series formed by the notes of the triad. It was thus important to ask if the general pattern of results obtained in the first experiment could be obtained for other notes in the triad that were not octave equivalents of a missing fundamental and hence could only be perceived analytically.

To this end, the standard stimuli for the next experiment were the same root-position and first-inversion A-major triads as were used in Experiment 1. However, instead of only adjusting pure tones in the frequency range of the root note of the triad, subjects were asked to adjust pure tones in the ranges of each of the notes of the triads.

\section{Method}

Subjects. The subjects were 33 undergraduate students, ranging in age from 17 to 45 years. Six male students and 7 female students were musically untrained, according to the criterion of Experiment 1 , whereas 7 male students and 13 female students were musically trained.

Apparatus and Stimuli. The apparatus was the same as that for the previous experiment. Standard stimuli were the same rootposition (A-880, C\#-1100, and E-1320 Hz) and first-inversion $(C \#-550, E-660$, and A-880 Hz) A-major triads as were used in Experiment 1. Comparison stimuli were pure tones whose initial frequencies were random within $\pm 1 \%$ to $\pm 3 \%$ of the frequency of one of the three notes of the standard triad. The subjects could then adjust the frequency of the comparison stimulus $\pm 6 \%$ of its initial value. The durations and intensities of the stimuli were the same as in the previous experiment.

Procedure. Each subject completed a total of 48 method-ofadjustments trials in a single session lasting approximately $1 \mathrm{~h}$. In a randomly selected half of the trials, the root-position triad was the standard stimulus, and in the other half, the first-inversion triad was the standard. On any one trial, adjustment of the comparison stimulus was restricted to vary approximately \pm 1 semitone about the frequency of one of the three notes in the standard triad. The various comparison-stimulus ranges were randomly and equally distributed over trials, so that there was a total of eight trials with each combination of triad position and comparison-stimulus range. Other aspects of the procedure were the same as for Experiment 1. 


\section{Results and Discussion}

Stimulus exposure. The subjects listened to a mean of 15.1 standard-comparison pairs per trial. This mean is slightly larger than that obtained in Experiment 1, which may reflect an increase in task difficulty owing to the variability across trials as to which triad pitch was relevant to adjusting the comparison stimulus. Analysis of variance of this measure indicated no significant effects involving musical training ( $F \mathrm{~s}<1.0$ ), although there was a slight tendency for the number of pairs per trial to increase as the frequency range of the comparison stimulus increased $[F(5,155)=3.00, p=.013]$.

Relative errors. Figure 1 shows the mean relative errors for each of the groups and trial types. As in the previous experiment, musically trained subjects were more consistent in their settings of comparison stimuli than were untrained subjects $[F(1,31)=22.70, p<.001]$. There was also a significant effect of trial type $[F(5,155)=5.65$, $p<.001]$, which resulted from more consistent settings when the comparison stimulus was restricted to the frequency range of the lowest note in the triad. There was also a marginally significant interaction of these two factors $[F(5,155)=2.29, p=.048]$, resulting from the greater consistency for the lowest note in the triad being most pronounced for the root-position triad in musically trained subjects, and for the first-inversion triad in the untrained subjects. This greater consistency during adjustment of a comparison stimulus in the frequency range of the lowest note in a triad may be related to Stumpf's (1883) finding that the pitch of the lower frequency component of a dyad is dominant or more easily "heard out."

Constant errors. Figure 2 shows the mean constant errors for each of the groups and trial types, referenced to the note of the triad whose frequency was contained within the range of the comparison stimulus. Constant errors were positive for frequency adjustments in the range of the lowest note of the standard triad, and they

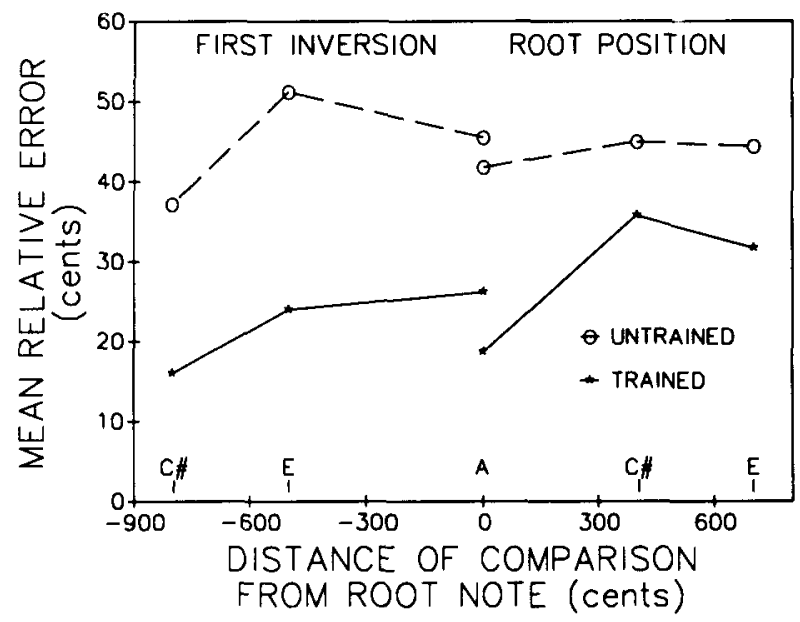

Figure 1. Mean relative errors in cents for the two musical training groups on the various combinations of standard and comparison stimuli in Experiment 2. For ease of interpretation, the name of the triad note contained within the range of adjustment of the comparison stimulus has been indicated on the abscissa.

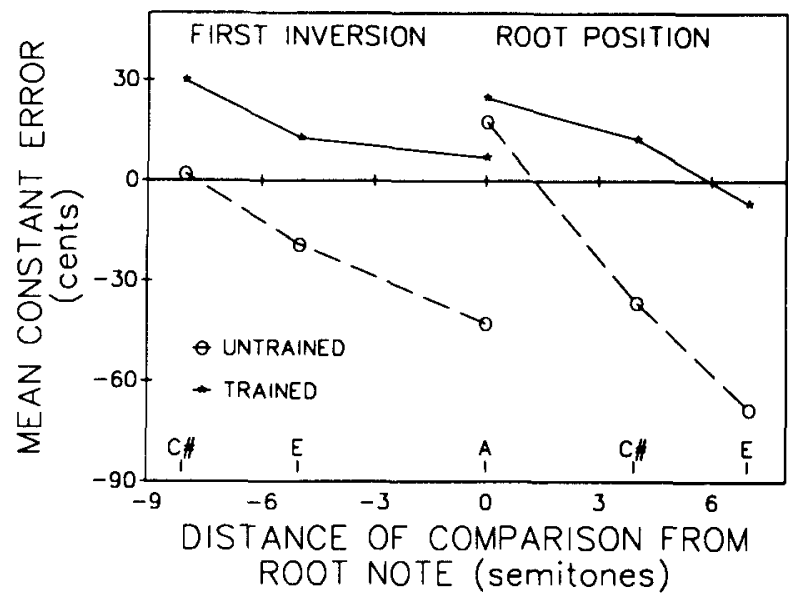

Figure 2. Mean constant errors in cents, referenced to the note of the triad contained within the range of adjustment of the comparison stimulus, for the two musical training groups on the various combinations of standard and comparison stimuli in Experiment 2. For ease of interpretation, the name of the triad note contained within the range of adjustment of the comparison stimulus has been indicated on the abscissa.

moved in a progressively negative direction for higher notes $[F(5,155)=29.57, p<.001]$. This effect was more pronounced for untrained subjects $[F(5,155)=5.88$, $p<.001]$, whose settings were more negative overall $[F(1,31)=29.18, p<.001]$. This pattern of constant errors in which the comparison stimulus was set relatively flatter as its frequency range varied from the lowest to the highest note in the standard triad extends the pitch interactions observed in Experiment 1 from the root note of the triad to all of its notes, thus showing that such interactions are not restricted to pitches that could be based on synthetic perception of a missing fundamental.

There is, however, one difference between the present constant errors and those found in the previous experiment. In Experiment 1, pitch interactions were only found for musically untrained subjects. In Experiment 2, musically trained subjects also showed such interactions, although to a much lesser extent than untrained subjects did. This difference can reasonably be attributed to the greater difficulty of analytically isolating the relevant pitch from the triad when it varies from trial to trial. However, the means in Figure 2 are misleading with respect to the performance of individual subjects. Some trained subjects showed no pitch interactions, whereas others showed substantial ones. All untrained subjects displayed pitch interactions, but the constant errors for some were much more negative overall than they were for others. To objectify these descriptions of homogeneous subgroups, a $K$ means cluster analysis was performed on the constant error data, using MICRO-CLUSTER (Edmonston, 1984) to find an optimal least-squares grouping of the subjects into four clusters.

Figure 3 shows the mean constant errors on the various trial types for each of the four resulting homogeneous groups of subjects. Cluster 1 consisted of 7 musi- 


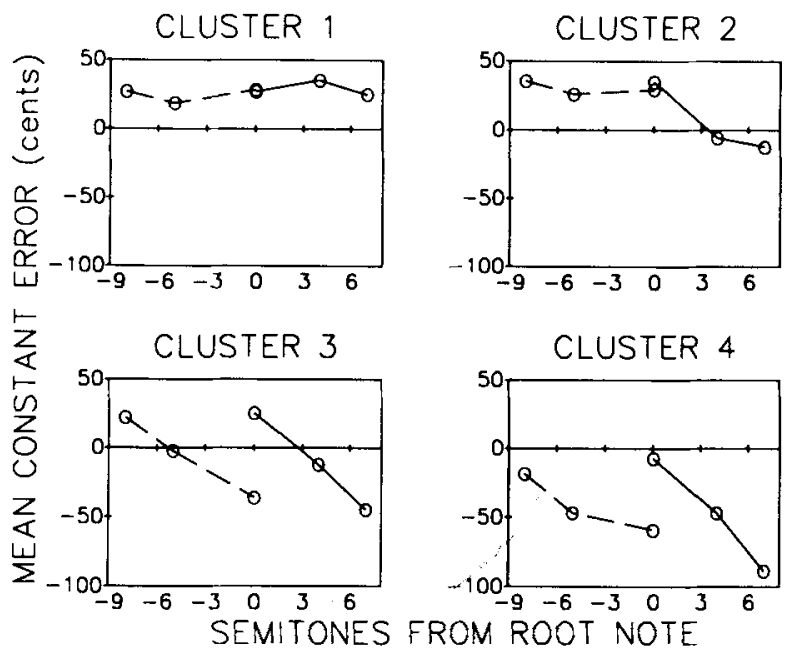

Figure 3. The constant errors of Figure 3 plotted separately for each homogeneous subject group, as determined by cluster analysis.

cally trained subjects who showed no pitch interactions. Cluster 2 consisted of 6 trained subjects and 1 untrained subject, who showed no pitch interactions for the firstinversion triad, but small ones for the root position. Thus, two-thirds of the trained subjects showed little or no pitch interaction, in spite of the increased variability in which note of the triad was relevant to adjusting the comparison stimulus. Cluster 3 was composed of 7 trained and 6 untrained subjects, who showed substantial pitch interactions for both triad positions. Cluster 4 consisted of the remaining 6 inexperienced subjects, whose performance was similar to that of those in Cluster 3 , except that their constant errors were more negative overall. Thus, essentially all of the untrained subjects and about one third of the trained subjects showed substantial and comparable pitch interactions. The only difference between the results for Clusters 3 and 4 was that those for Cluster 4 were shifted negatively. It could be that different component pitches were dominant for different subjects. Cluster 4 subjects may have been most strongly influenced by, or could most easily "hear out," the lowest note of the triad, whereas Cluster 3 subjects were most strongly affected by the middle note, or by all three equally.

\section{EXPERIMENT 3}

The two experiments just reported demonstrate a tendency among subjects to accommodate a comparison stimulus to all the pitch components of a triad when attempting to match it to any particular component. The strength of this tendency decreases rapidly with musical training, ultimately allowing adjustments that are free of pitch interactions between component notes and hence of dependence on the position of the triad. These results raise the possibility that such pitch interactions are determined not only by the placements of the various triad components on the frequency dimension, but also by the salience of the var- ious components. Indeed, individual differences, such as those obtained between Clusters 3 and 4 in Experiment 2, appeared to be explicable in terms of different subjects' finding one or another component note of the triad to be more salient than the others and hence producing larger effects on the setting of the comparison stimulus.

In the final experiment of this series, Experiment 3, we sought more objective evidence for the possibility that the pitch interactions demonstrated in the previous experiments depended on the relative salience of the component notes in the triad. The salience of a particular note was manipulated by varying its intensity relative to that of the other notes in the triad. The standard stimuli for this experiment were root-position and first-inversion A-major triads, but in some instances either the highest or lowest note in the triad was made more salient by increasing its relative intensity. If the salience of the component notes in the triad are important to determining pitch interactions between them, we would expect comparison-stimulus settings to be more displaced in the direction of a higher intensity note.

A subsidiary purpose of this third and final experiment was to extend the generality of the pitch interactions found in the first two experiments to a pitch range more typical of musical materials and to more noise-free stimuli. The triads used in Experiments 1 and 2 were constructed around A-880 Hz, which is a relatively high pitch for most common musical instruments. In Experiment 3, the pitch range was reduced by an octave to be more typical of musical materials. Also, the stimuli were generated by equipment that allowed a much better signal-to-noise ratio. Because of these procedural changes, care was taken to design the experiment so as to include a systematic replication (Sidman, 1960) of the pitch interactions found in Experiment 1.

\section{Method}

Subjects. Twenty-five subjects, ranging in age from 18 to 28 years, participated in Experiment 3. The subjects were divided into three groups on the basis of their self-reported musical training. The untrained group consisted of 9 women and 2 men with no formal musical training. The trained group consisted of 5 women and 3 men with a minimum of 3 years of formal musical training. The mean duration of formal musical training in this group was 9.9 years. A professional group consisted of 4 women and 2 men who were graduates of, or senior students in, a university music program. These subjects had a mean of 15.5 years of formal musical training.

Apparatus. The experiment took place in an AEC soundattenuating room. Stimuli were generated by a DMX-1000 signalprocessing computer, slaved to a LSI-11/23 computer that scheduled stimuli and recorded responses. The DMX-1000 uses 16-bit DACs driven at a sampling rate of $19.3 \mathrm{kHz}$ into a $9.6-\mathrm{kHz}$ lowpass filter for smoothing. The resulting signal-to-noise ratio is $96 \mathrm{~dB}$. Auditory stimuli were conditioned by a Model Z501 Avance DC Power Amplifier and presented binaurally at $72 \mathrm{~dB}$ SPL through Realistic Pro IIA earphones. Subjects used a 10-turn potentiometer to adjust the frequency of comparison stimuli with a resolution of $0.3 \mathrm{~Hz}$.

Stimuli. The standard stimuli for this experiment were justly intoned, A-major triads in root position and first inversion. The triads were composed of pure tones with the root-position triad consisting of $\mathrm{A}-440, \mathrm{C}-550$, and $\mathrm{E}-660 \mathrm{~Hz}$. The first-inversion triad con- 


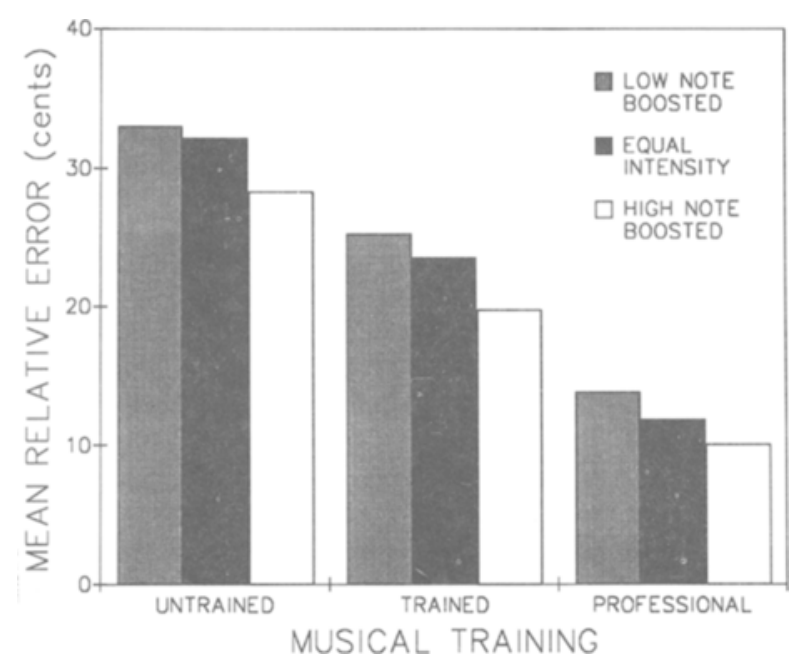

Figure 4. Mean relative errors in cents for the three subject groups in Experiment 3, as a function of which, if any, note of the standard triad was boosted in intensity.

sisted of C\#-275, E-330, and A-440 Hz. These triads were voiced an octave lower than those in the first two experiments, in an attempt to generalize results to a frequency range more typical of musical scores.

On some trials, the highest or lowest note of a triad was emphasized by increasing its intensity relative to that of the other two notes. This was accomplished by increasing the intensity of that note $12 \mathrm{~dB}$, and then reattenuating the entire triad to a level of $72 \mathrm{~dB} \mathrm{SPL}$. The increase in loudness of the emphasized note was clearly audible, but it was not sufficient to completely mask the other two notes. The comparison stimulus was always a pure tone with an initial value that varied randomly within $\pm 1 \%$ to $\pm 3 \%$ of $A-440 \mathrm{~Hz}$, and could be adjusted over a range of $\pm 6 \%$ of its initial value.

Procedure. Each subject completed 48 method-of-adjustments trials in a single session lasting approximately $1 \mathrm{~h}$. Each trial began with the subject centering the potentiometer knob with the aid of a numeric display on a video monitor. The standard triad was then presented for $0.5 \mathrm{sec}$, followed by $0.5 \mathrm{sec}$ of silence, and then $0.5 \mathrm{sec}$ of the pure-tone comparison. After $1.0 \mathrm{sec}$ of silence, this stimulus sequence was repeated until the subject was satisfied that the comparison had been adjusted to sound maximally similar to the standard. As in the previous two experiments, adjustments could be made at any time, but this did not affect the comparison stimulus until its next onset.

Each of the two triad positions served as standard stimuli on a random half of the trials. On a random one third (eight) of the trials for each triad position, the intensities of all three triad notes were equal. On another third, the relative intensity of the highest note in the triad was increased, and on the remaining third of the trials, the relative intensity of the lowest note in the triad was increased.

\section{Results}

Stimulus exposure. The subjects listened to a mean of 11.0 standard-comparison pairs per trial when the A$440 \mathrm{~Hz}$ note of a triad was more intense than the other notes. When the notes were of equal intensity or another note was more intense than the $\mathrm{A}$, this mean increased to 14.6. In an analysis of variance, this difference produced a significant interaction of triad position and whichever, if any, note of the triad was increased in intensity $[F(2,44)=17.27, p<.001]$. It thus appears that the subject's task was somewhat easier when the note of the triad corresponding to the comparison stimulus was more salient. There were no significant effects of musical training on number of standard-comparison pairs.

Relative errors. Figure 4 shows the mean relative errors for the various groups as a function of what, if any, note of the standard triad was emphasized by an increase in intensity. An analysis of variance indicated that consistency of comparison-stimulus settings again increased with musical training $[F(2,22)=7.4, p<.05]$. There was also a significant main effect of which, if any, note of the triad was increased in intensity $[F(2,44)=3.47$, $p<.05]$. This effect resulted from an increase in consistency of comparison settings when the highest note in the triad was increased in intensity, and a slight reduction in consistency when the lowest note was emphasized. Triad position had no significant effect, and there were no significant interactions.

Constant errors. Figure 5 shows the mean constant errors, relative to $A-440 \mathrm{~Hz}$, for each of the groups and standard triads. As in the first two experiments, the com-
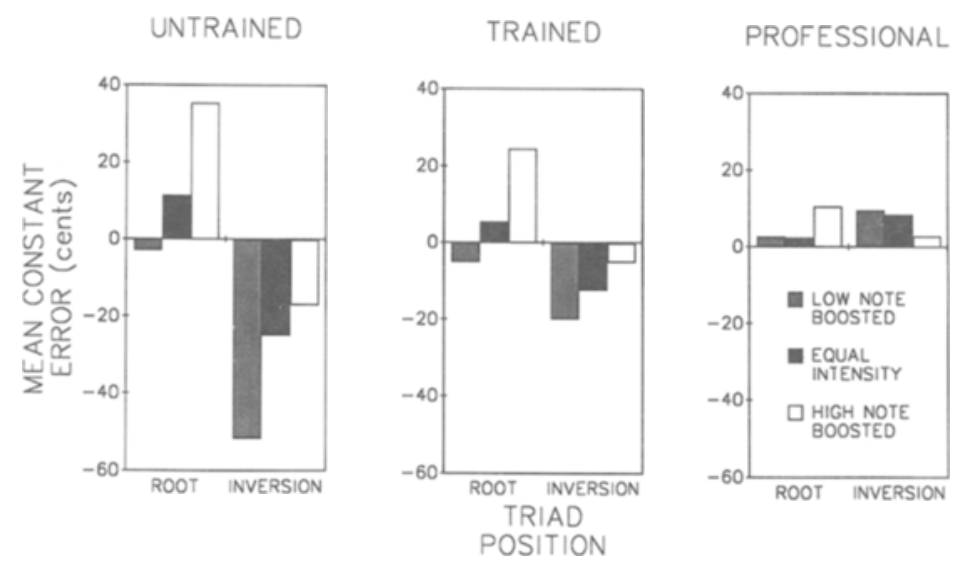

Figure 5. Mean constant errors in cents, referenced to the root note of the standard triad, for the two musical training groups and six standard triads in Experiment 3. 
parison stimulus was set flatter for the first-inversion triad, in which the other notes were of lower frequency, and sharper for the root-position triad, in which the other notes were of higher frequency $[F(1,22)=15.97, p<.001]$. The magnitude of these pitch interactions decreased with level of musical training $[F(2,22)=6.55, p=.006]$, so that they were smaller for trained than for untrained subjects, and totally absent for professional subjects who displayed small positive constant errors for both triad positions. These results show that the pitch interactions obtained in the first experiment were in no way dependent on the relatively high-pitched and noisy stimuli used in the first two experiments.

The results of main interest in Experiment 3 were the effects of emphasizing the highest or lowest note in the standard triad by increasing its intensity. Comparison settings were displaced in the direction of the emphasized note $[F(2,44)=15.49, p<.001]$, and the magnitude of this displacement decreased as level of musical training increased $[F(4,44)=4.27, p=.005]$, being totally absent in professional subjects. This displacement also interacted with triad position $[F(2,44)=7.66, p=.001]$, because emphasizing the triad's root note, which was within the range of adjustment of the comparison stimulus, produced a small reduction in constant error, whereas emphasizing a note other than the root produced a large increase in constant error. These results clearly indicate that the pitch interactions under examination in the present studies were determined both by the placement of the various triad notes on the frequency dimension and by the relative salience of these notes.

\section{GENERAL DISCUSSION}

The experiments reported in this paper were designed to examine whether the analytically perceived pitch of a component note of a major triad is in any way dependent on the other notes present, and hence, on the position of the triad. When musically untrained subjects were asked to adjust the frequency of a pure-tone comparison stimulus over a range restricted to the pitch class of the root note of a major triad until it sounded maximally similar to the triad, the comparison-stimulus settings deviated from the root note in the direction of the other notes in the triad. Musically trained subjects always set the comparison stimulus slightly sharper than the root note of the triad, but showed no such pitch interactions.

Experiment 2 generalized these results from comparison stimuli restricted to the frequency range of the root note of a triad to ranges corresponding to each of the triad's component notes. This shows that the results of the first two experiments were based on analytic perception of the component notes of the triads, and not dependent on a synthetically perceived missing fundamental, since the nonroot notes of a major triad can only be perceived analytically.

The last experiment in this series showed that these pitch interactions are influenced not only by the position of the various triad notes on the frequency dimension, but also by the relative salience of the individual notes. In addition, in the last two experiments, which probably made analytic perception of a particular triad note more difficult because of increased uncertainty about which note was relevant or because of variations in the salience of individual notes, pitch interactions were found for some musically trained subjects. This indicates that even musically trained subjects are not immune to such effects if the analytic listening task is sufficiently difficult.

There appear to be two possible mechanisms for the pitch interactions displayed by the musically less trained subjects in the present experiments. The successive presentation of a standard triad, followed by a pure-tone comparison stimulus, required the subjects to extract pitch information from the triad and retain it for subsequent comparison with the pure tone. One possibility is that the extracted pitch percepts corresponding to the individual notes of the triad were not independent of each other. There is indeed evidence that the primary pitch of a pure tone is shifted slightly ( $1 \%$ or $2 \%$ ) by the addition of a pure tone of a different frequency (e.g., Plomp, 1976; Terhardt, 1971). However, the direction of this pitch shift increases the apparent interval between the two pitches, rather than decreases it, as would be required to explain the present pitch interactions.

A more likely explanation of the present pitch interactions would thus operate at the point in time at which a subject attempted to match the comparison stimulus to the various pitch percepts extracted from the standard triad. It would appear that musically less trained subjects were unable to base this match solely on the extracted pitch contained within the range of the comparison stimulus, thus accommodating their comparison stimulus settings slightly to the pitches of the other notes of the triad, or to some more abstract impression of the overall pitch height of the triad. Such a phenomenon would suggest that considerable caution should be employed in using adjustment of a pure tone to measure the primary or spectral pitch of a particular component of a complex tone. At the very least, the subjects should be highly musically trained or should receive specialized training in such analytic hearing.

From a musical perspective, these results show an interesting correspondence between levels of musical training and analytic listening skills. When asked to listen to a major triad, and then to adjust a pure tone to match one of its component notes, musically untrained subjects did not appear to be able to completely isolate this pitch from others present in the triad. As a result, their adjustments of a comparison stimulus corresponding to a particular note of the triad were not independent of triad position. Subjects with greater amounts of musical training, on the other hand, were able to match the comparison to any particular note in the triad without being affected by the triad's position. This improvement in analytic listening is probably a result of musical training, although on the basis of the present experiments, one cannot reject the 
possibility that subjects with better analytic listening skills may be more likely to become musically trained. In either case, this improved analytic listening across levels of musical training could be related to realistic musical skills, such as the ability to sing or play in tune with a melody embedded in a harmonic accompaniment.

Any direct application of these results to music perception should obviously await replication with musically valid stimuli. We have run a few subjects in the paradigm of Experiment 1 using equal temperament and harmonically rich, sawtooth tones. The results were essentially identical to those of Experiment 1, but a more complete replication of the present work with such stimuli is needed.

\section{REFERENCES}

EdMONSTON, B. (1984). MICRO-CLUSTER: Cluster analysis software for microcomputers. Journal of Classification, 2, 127-130.

GreER, R. D. (1970). The effect of timbre on brass-wind intonation. In E. Gordon (Ed.), Experimental research in the psychology of music (pp. 65-94). Iowa City: University of lowa Press.

Helmholtz, H. L. F. von (1863). Die Lehre von den Tonempfindungen als Physiologische Grundlage fur die Theorie der Musik. Braunschweig: F. Vieweg.

Krumhansl, C. L., Bharucha, J. J., \&e Kessler, E. J. (1982). Perceived harmonic structure in three related musical keys. Journal of Experimental Psychology: Human Perception \& Performance, 8, 24-36.

LICHTE, W. H. (1941). Attributes of complex tones. Journal of Experimental Psychology, 28, 455-465.

PLATT, J. R., \& RACINE, R. J. (1985). Effect of frequency, timbre, experience, and feedback on musical tuning skills. Perception \& Psychophysics, 38, 543-553.
Platt, J. R., Racine, R. J. (in press). Perceived pitch class of isolated musical triads. Journal of Experimental Psychology: Human Perception \& Performance.

Plomp, R. (1964). The ear as a frequency analyzer. Journal of the Acoustical Society of America, 36, 1628-1636.

Plomp, R. (1976). Aspects of tone sensation. New York: Academic Press.

Pollack, I. (1964). Ohm's acoustical law and short-term auditory memory. Journal of the Acoustical Society of America, 36, 2340-2345.

Rameau, J. P. (1971). Treatise on harmony. New York: Dover. (Original work published 1750 )

Roberts, L. A., \& Shaw, M. L. (1984). Perceived structure of triads. Music Perception, 2, 95-124.

Sidman, M. (1960). Tactics of scientific research. New York: Basic Books.

SPIEGel, M. F., WAtson, C. S. (1984). Performance on frequencydiscrimination by musicians and nonmusicians. Joumal of the Acoustical Society of America, 76, 1690-1695.

STUMPF, C. (1883). Tonpsychologie. Leipzig: Hirzel.

TERHARDT, E. (1971). Pitch shifts of harmonics, an explanation of the octave enlargement phenomenon. Proceedings of the 7 th International Congress of Acoustics, 3, 621.

TERHARDT, E. (1974). Pitch, consonance and harmony. Joumal of the Acoustical Society of America, 55, 1061.

TERHARDT, E. (1984). The concept of musical consonance: A link between music and psychoacoustics. Music Perception, 1, 276-295.

Terhardt, E., \& GruberT, A. (1987). Factors affecting pitch judgments as a function of spectral composition. Perception \& Psychophysics, 42, 511-514.

Terhardt, E., Stoll, G., Seewann, M. (1982). Algorithm for extraction of pitch and pitch salience from complex tonal signals. Journal of the Acoustical Society of America, 71, 679-688.

(Manuscript received July 13, 1989; revision accepted for publication January 22, 1990.) 